\title{
Endotracheal intubation skills of pediatricians versus anesthetists in neonates and children
}

\author{
Sam J. van Sambeeck ${ }^{1}$. Sander M. J. van Kuijk ${ }^{2}$ - Boris W. Kramer ${ }^{1} \cdot$ Petronella M. Vermeulen $^{3} \cdot$ Gijs D. Vos ${ }^{1}$
}

Received: 15 November 2018 /Revised: 5 March 2019 / Accepted: 30 April 2019 /Published online: 8 June 2019

(C) The Author(s) 2019

\begin{abstract}
This study compares the performance of pediatricians and anesthetists in neonatal and pediatric endotracheal intubations (ETI) during simulated settings. Participants completed a questionnaire and performed an ETI scenario on a neonatal and a child manikin. The procedures were recorded with head cameras and cameras attached to standard laryngoscope blades. The outcomes were successful intubation, time to successful intubation, number of attempts, complications, total performance score, endassessment rating, and an assessment whether the participant was sufficiently able to perform an ETI. Fifty-two pediatricians and 52 anesthetists were included. For the neonatal ETI, the rate of successful intubation was in favor of anesthetists although not significant. Anesthetists performed significantly better in all other outcomes. Of the pediatricians, $65 \%$ was rated sufficiently adept to perform a neonatal ETI vs $100 \%$ of the anesthetists. Pediatricians (29\%) overestimated while anesthetists (33\%) underestimated their performance in neonatal ETI. For the pediatric ETI, all outcomes were significantly better for anesthetists. Only $15 \%$ of all pediatricians were considered sufficiently able to perform pediatric ETI vs $94 \%$ of the anesthetists.

Conclusion: Anesthetists are far more adept in performing ETI in neonates and children compared with pediatricians in a simulated setting. Complications are expected to occur less frequently and less seriously when anesthetists perform ETI.

What is Known:

- Endotracheal intubation (ETI) performed by inexperienced care providers can lead to unsuccessful and/or prolonged intubation attempts. This can cause complications such as hypoxemia, trauma to the oropharynx and larynx, and prolonged interruption of resuscitation, which results in a high morbidity/mortality.

- Fifty to 60 real-life ETI procedures are needed before ETI can be performed with a 90\% success rate. Despite this, $18 \%$ of providers still require some assistance even after performing 80 intubations. Skill fade will occur if there is too little exposure.

What is New:

- This study shows that, on both neonatal and child manikins, anesthetists perform better in ETI compared with pediatricians. Besides this, complications are expected to occur less frequently and less seriously when anesthetists are performing the ETIs on neonates and children.

- In those countries where there are no clear interprofessional agreements made in general hospitals on who will perform ETI on neonates and children in acute care settings, these agreements are urgently necessary.
\end{abstract}

Keywords Child $\cdot$ Infant $\cdot$ Newborn $\cdot$ Endotracheal intubation $\cdot$ General hospitals

\section{Abbreviations}

APLS Advanced Pediatric Life Support

EPALS European Pediatric Advanced Life Support
ETI Endo-tracheal intubation

NLS Neonatal Life Support
Communicated by Piet Leroy

Electronic supplementary material The online version of this article (https://doi.org/10.1007/s00431-019-03395-8) contains supplementary material, which is available to authorized users.

Sam J. van Sambeeck

vansambeeck@gmail.com

Extended author information available on the last page of the article

\section{Introduction}

Endotracheal intubation (ETI) is the golden standard for securing the airway in situations where the provider is unable to ventilate the patient adequately with a bag-and-mask or by a supraglottic airway device, or if an open airway is compromised [20,24]. Unsuccessful intubation attempts lead to complications, resulting in a high morbidity/mortality rate $[1,2$, $18,21]$. There is a direct correlation between the experience of 
the ETI provider and the success rate, intubation time, and number of attempts needed for each ETI [3, 9, 13, 22, 25]. In Dutch general hospitals, the attending pediatrician is responsible for the acute care of critically ill neonates and children, sometimes in co-operation with emergency physicians, anesthetists, intensivists, and nursing staff [14]. In Dutch general hospitals, there is no pediatric intensive care specialist available who can perform pediatric ETI in acute settings. In these hospitals, due to the low incidence of critically ill children and newborns requiring an acute ETI $[7,11,17]$, the pediatricians' exposure to ETI is expected to be low [14, 17]. Our hypothesis is that, since anesthetists have more experience in ETI, they have better intubation skills and higher success rates than pediatricians in ETIs. The study objectives are (1) to explore the actual exposure of general pediatricians and anesthetists to ETI in neonates and children; (2) to compare the intubation skills (success rate, intubation time, number of attempts, degree of laryngeal view, complications, and overall performance) of both groups in a neonatal and a child manikin setting; and (3) to compare the self-perceived capability of the ETI performance with the actual performance on the manikins.

\section{Materials and methods}

\section{Study design}

A cross-sectional study was performed among pediatricians and anesthetists, practicing in general hospitals. At their respective annual national medical conferences, specialists were asked to volunteer to perform ETI procedures on a neonate and a child manikin. Exclusion criteria for participants were (1) working in a tertiary facility or university hospital, (2) not participating in neonatal care or acute care of critically ill children, and (3) not participating in on-call duties.

The research and scenario setup were standardized for all settings. All participants started with an electronic survey (Appendix A), after which they continued with the intubation scenarios.

\section{Procedures}

The electronic survey consisted of a general section concerning age and time since completion of residency, a second section concerning exposure to ETI in neonates and children in the past year, and their self-perceived competence. Also, participants were asked their opinion about which medical specialist would be the most suited to perform an ETI on neonates and children. General questions were multiple choices. Questions concerning their self-perceived competence or opinions were based on a five-point Likert scale $(1=$ completely incompetent to $5=$ highly competent $)$ and
( $1=$ not at all preferred to $5=$ very preferred $)$. The questionnaires were anonymous, coded per specialty, and linked to the intubation performances on both manikins.

After completing the survey, the participant was equipped with a head camera (Go Pro Hero4 Silver ${ }^{\circledR}$, San Mateo, USA) and proceeded to the intubation scenario on the neonatal and child manikin. The setup of both manikins was identical, apart from size. Participants received information about the manikins' age and clinical condition. They were asked to perform an ETI by direct laryngoscopy on the neonatal and child manikins, just as they would perform it in real-life situations. The intubation procedures were filmed in overview by the head camera. The view from the laryngoscope blades was filmed with 5 -mm cameras with lighting (Waterproof Endoscope Camera ${ }^{\circledR}$ USB $5 \mathrm{~mm}$ 6LED, J\&S United Technology, Taipei, Taiwan), attached to standard laryngoscope blades (Macintosh and Miller), replacing the original light source. The laryngoscope blades and corresponding handles were similar to those used in daily clinical practice. The two manikin scenarios were (1) neonatal manikin (Newborn Anne, Laerdal Medical ${ }^{\circledR}$, Stavanger, Norway), representing a fullterm newborn female, weight $3500 \mathrm{~g}$. She was born in the delivery room after an uncomplicated pregnancy. At birth, there was no spontaneous breathing after five sustained insufflation breaths. The circulation was normal. (2) Child manikin (SimJunior, Laerdal Medical®, Stavanger, Norway), representing a 6-year-old previously healthy boy admitted to the emergency department with acute respiratory insufficiency and secondary apnea, but with normal circulation. The manikin was placed in supine face straight-up position, the table height was $78 \mathrm{~cm}$. To perform the intubation, participants could use different laryngoscope blades (Miller size 1 and Macintosh size 1-4), endotracheal tubes (sizes 2.5-6.5, cuffed and uncuffed), Magill forceps (size 7 child and 9 adult), and stylet. Bag-valve-mask resuscitator $(500 \mathrm{ml}$ and $1600 \mathrm{ml})$ and masks (sizes 0-5) were available to perform bag-mask ventilation (Laerdal Silicone Resuscitator, Laerdal Medical®, Stavanger, Norway). There was one assistant available per station, who could reach for materials when asked for. There was no feedback of vital signs or patient status given during the procedure.

\section{Outcomes}

Three experts, blinded for the specialty of the participants, individually rated all the videos of the performances of the participants in different orders. These experts, further referred to as observers, consisted of a senior pediatric-intensivist, a senior neonatologist, and a senior pediatric-anesthetist, all working in a tertiary facility university medical center and with extensive experience in airway management. All observers rated the intubation procedures of the participants independently, using the footage from the overview head 
camera and the laryngoscope blade cameras. A predefined 8item scoring list (Appendix B) was used to rate the intubation performance, further referred to as the total performance score. Higher scores indicate better performance; positive and negative ratings could be given to different components, with a maximum total score of 13 points. This total performance score was based on (1) steps outlined in the Advanced Pediatric Life Support (APLS) and European Pediatric Advanced Life Support (EPALS) airway management checklists and (2) expert opinion by the observers.

The primary outcome of this study was the intubation success rate defined as an endotracheal tube placed through the vocal cords. Secondary outcomes included the time to successful intubation, the number of attempts, degree of laryngeal view, the number of complications (laryngoscope blade between the vocal cords, esophagus intubation, transferring the laryngoscope handle from one hand to the other during intubation and incorrect cuff placement (between the vocal cords)), and the total performance score (see Appendix B). In addition, an end-assessment rating from 1 to 10 (1 being the lowest and 10 being the highest score) and the impression whether the participant was sufficiently capable to perform a safe ETI in a neonate and a child were secondary outcomes.

\section{Statistical analysis}

Characteristics of the participants were reported as absolute values and percentages, stratified by specialty. Differences between pediatricians and anesthetists were tested using Pearson's chi-squared test.

For both manikins, the outcome measures of the total performance score and the end-assessment grade between pediatricians and anesthetists were tested using the Mann-Whitney $U$ test. Differences in the proportion of participants that performed successful ETI and the proportion of participants that were found sufficiently able to perform the procedures were tested using Pearson's chi-squared statistic. In case of expected cell counts of five or less, we used Fisher's exact test. The difference between pediatricians and anesthetists on the number of attempts needed for successful ETI was tested using the non-parametric Mann-Whitney $U$ test, while the difference in total time required to perform ETI was tested using the independent $t$ test.

In case of disagreement between observers on categorical scales, the category that was scored by the majority was used for the analysis. Otherwise, the average score was used for the analysis for continuous items. We used Cohen's kappa to determine agreement between observers for binary items, and the intra-class correlation coefficient (ICC) for (semi-) continuous variables. In case of perfect agreement on a binary item, only the total agreement was computed. Self-reported clinical experience and the self-perceived capability of the participants are tested for differences between pediatricians and anesthetists using Pearson's chi-squared test. All analyses were performed using IBM (New York, USA) SPSS version 23. Figures were made in R version 3.3.3 (Vienna, Austria).

\section{Results}

Out of 132 participants, 104 were eligible for analysis. Twenty-eight (21.1\%) participants were excluded because of incomplete survey, incorrect instructions, no on-call duty, or video/camera error (e.g., incomplete view). Out of these 104 participants, 52 were registered pediatricians and 52 registered anesthetists.

\section{Electronic survey}

Characteristics of the participating physicians are shown in Table 1 . There were no statistically significant differences in age or length of time since completion of residency between both groups. Questionnaire responses of the participants about existing agreements on who is performing ETI in neonatal and pediatric acute care settings and who preferably should perform ETI are shown in Table 1 as well. An online supplement (Table $5 \mathrm{a}$ and $5 \mathrm{~b}$ ) shows the pre-study ETI training of the participant in the past year.

Figure 1a shows the distribution of self-reported experiences with ETI of both groups' participants over the past year. On average, anesthetists reported to have performed pediatric ETI more often than pediatricians, not statistically significant for ETI in neonates $(p=0.738)$, but statistically significant for ETI in children $(p<0.001)$. Figure $1 \mathrm{~b}$ shows the distribution of self-perceived capability of performing ETI on neonates and children. The self-perceived capability of performing ETI in children was statistically significantly higher in anesthetists compared with pediatricians $(p<0.001)$.

\section{Endo-tracheal intubation performance}

Neonatal ETI We observed a non-significant difference in the proportion of pediatricians versus anesthetists that performed a successful neonatal ETI (i.e., tube in larynx through vocal cords) $(90.4 \%$ versus $100 \%, p=0.057)$. On all other scores, there was a significant difference in performance all in favor of the anesthetists.

Thirty-eight $(73.1 \%)$ pediatricians succeeded the ETI in one attempt, compared with $51(98.1 \%)$ of the anesthetists $(p=0.001)$. Figure 2 (upper half) shows the distribution of the time and number of attempts needed to perform an ETI per specialty. On average, pediatricians needed $47.7 \mathrm{~s}$ to perform ETI compared with $27.1 \mathrm{~s}$ by the anesthetists $(p<0.001)$. The median total performance score using the predefined scoring list (Appendix B) was 7.5 for the pediatricians compared with 11.5 for the anesthetists $(p<0.001)$. The median number of complications was 1.0 for the pediatricians versus zero for 
Table 1 Characteristics and questionnaire responses of all participants, stratified by specialty

\begin{tabular}{|c|c|c|c|}
\hline & Pediatricians $(n=52)$ & Anesthetists $(n=52)$ & $p$ value for difference \\
\hline \multicolumn{3}{|l|}{ Age } & \multirow[t]{5}{*}{0.138} \\
\hline$<40$ years & $8(15.4 \%)$ & $10(19.2 \%)$ & \\
\hline $40-50$ years & $32(61.5 \%)$ & $21(40.4 \%)$ & \\
\hline $51-60$ years & $7(13.5 \%)$ & $15(28.8 \%)$ & \\
\hline$>60$ years & $5(9.6 \%)$ & $6(11.5 \%)$ & \\
\hline \multicolumn{3}{|l|}{ Time since completion of residency } & \multirow[t]{5}{*}{0.162} \\
\hline$<5$ years & $6(11.5 \%)$ & $9(17.3 \%)$ & \\
\hline $5-10$ years & $11(21.2 \%)$ & $8(15.4 \%)$ & \\
\hline $11-20$ years & $27(51.9 \%)$ & $19(36.5 \%)$ & \\
\hline$>20$ years & $8(15.4 \%)$ & $16(30.8 \%)$ & \\
\hline \multicolumn{3}{|c|}{ Are there written agreements about who performs ETI in neonates and children? } & \multirow[t]{4}{*}{0.066} \\
\hline Yes & $11(21.2 \%)$ & $22(42.3 \%)$ & \\
\hline No & $15(28.8 \%)$ & $10(19.2 \%)$ & \\
\hline Do not know & $26(50.0 \%)$ & $20(38.5 \%)$ & \\
\hline \multicolumn{3}{|l|}{ Who is performing ETI in neonates and children? } & \multirow[t]{6}{*}{0.047} \\
\hline Pediatrician & $2(3.8 \%)$ & $11(21.2 \%)$ & \\
\hline Anesthetist & $29(55.8 \%)$ & $21(40.4 \%)$ & \\
\hline Pediatrician in neonates, anesthetist in children & $5(9.6 \%)$ & $2(3.8 \%)$ & \\
\hline Do not know & $12(23.1 \%)$ & $16(30.8 \%)$ & \\
\hline Otherwise ("most capable person") & $4(7.7 \%)$ & $2(3.8 \%)$ & \\
\hline \multicolumn{3}{|l|}{ Is it preferred that anesthetist perform the neonatal ETI? } & \multirow[t]{4}{*}{0.010} \\
\hline Not preferred & $22(42.3 \%)$ & $7(13.5 \%)$ & \\
\hline Neutral & $21(40.4 \%)$ & $31(59.6 \%)$ & \\
\hline Preferred & $9(17.3 \%)$ & $14(26.9 \%)$ & \\
\hline \multicolumn{3}{|c|}{ Is it preferred that anesthetist perform the pediatric ETI? } & \multirow[t]{4}{*}{0.030} \\
\hline Not preferred & $4(7.7 \%)$ & $5(9.6 \%)$ & \\
\hline Neutral & $9(17.3 \%)$ & $23(44.2 \%)$ & \\
\hline Preferred & $39(75.0 \%)$ & $24(46.1 \%)$ & \\
\hline
\end{tabular}

Fig. 1 a Self-reported experiences (on a yearly basis) with endotracheal intubation in neonates and children. b Selfperceived capability of performing endotracheal intubation on neonates and children

\section{a}

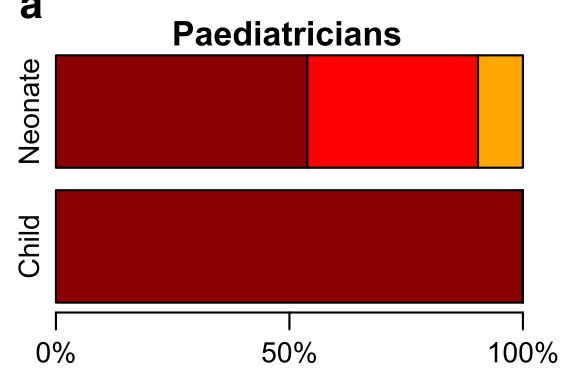

b

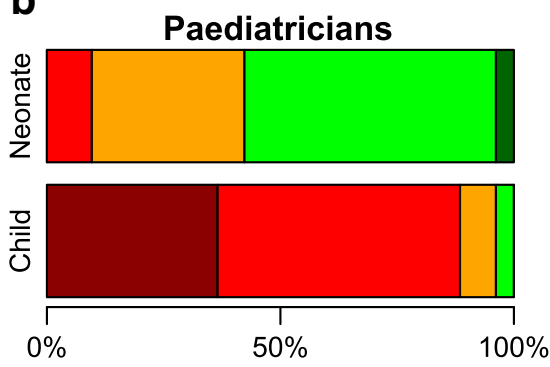

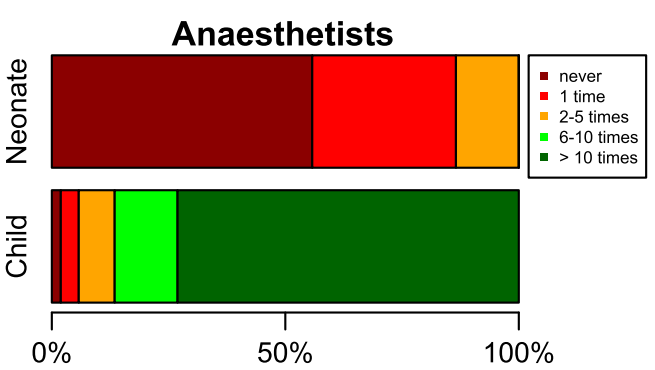

Anaesthetists

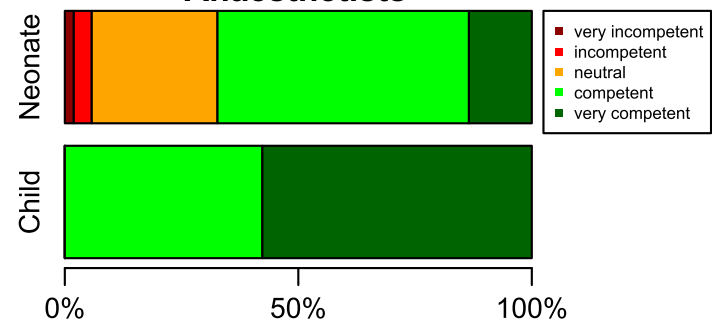



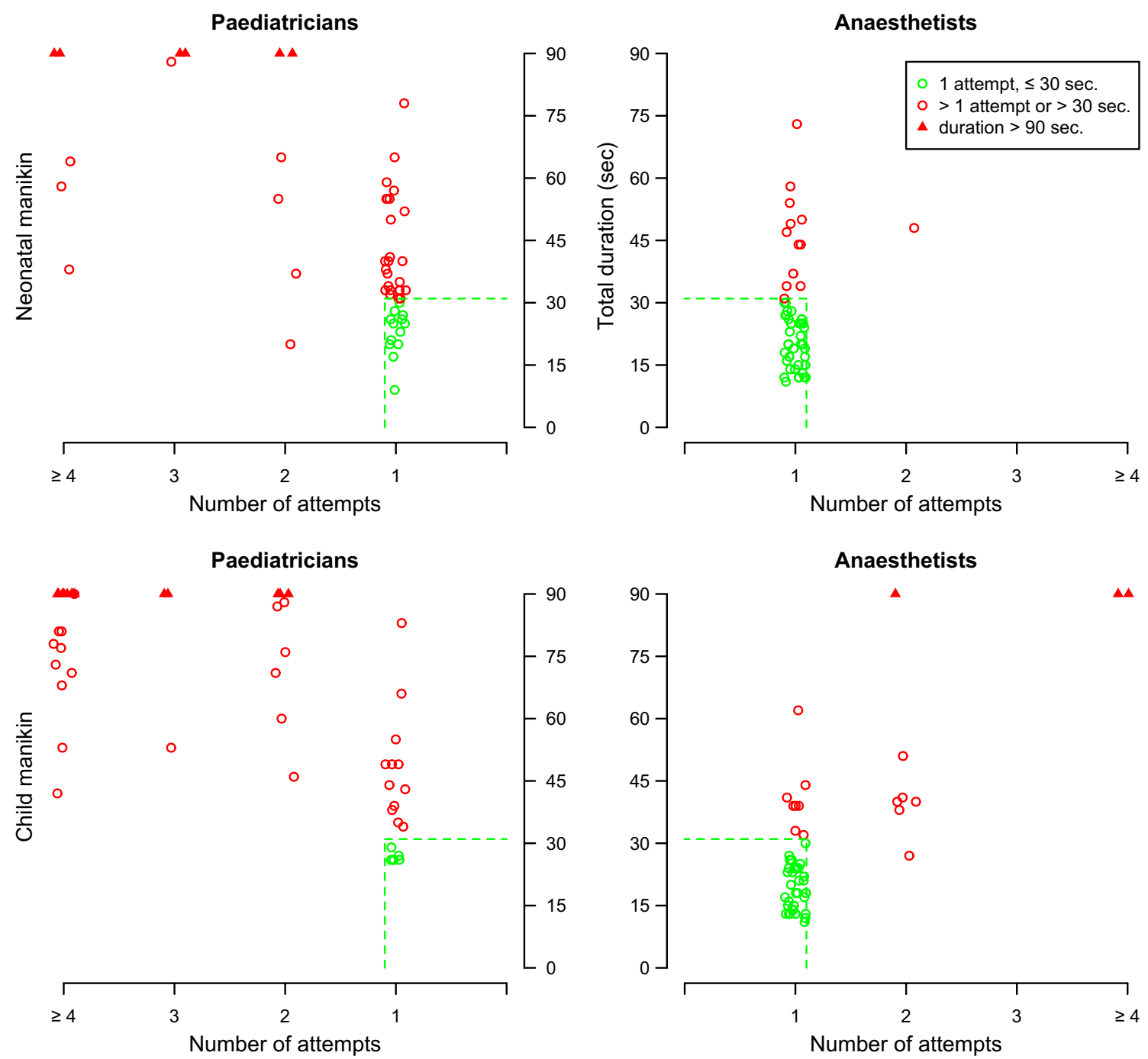

Fig. 2 Distribution of the time and number of attempts needed to perform ETI on both manikins per specialty (mirror wise)

the anesthetists $(p \leq 0.001)$. Pediatricians scored a significantly lower end-assessment grade compared with the anesthetists (median 5.8 versus $7.6, p<0.001$ ). For the neonatal manikin, $34(65.4 \%)$ pediatricians were considered sufficiently able to perform the procedure, compared with $52(100 \%)$ of the anesthetists $(p<0.001)$. Figure 3 shows the distribution of the endassessment grade, the total performance score, and the consideration "(in)sufficiently able to perform ETI" per specialty.

Pediatric ETI The difference in a successful pediatric ETI was statistically significant (pediatricians $57.7 \%$ versus anesthetists $96.2 \%, p<0.001)$. Eighteen $(34.6 \%)$ pediatricians succeeded in one attempt versus $43(82.7 \%)$ of the anesthetists $(p<0.001)$. Two pediatricians $(4 \%)$ refused to perform the procedure on the child manikin, since they felt not capable of performing the procedure and they would not perform this procedure in their hospital.

Figure 2 (lower half) shows the distribution of the time and number of attempts needed to perform a pediatric ETI per specialty. Pediatricians needed $83.4 \mathrm{~s}$ to perform ETI compared with $33.6 \mathrm{~s}$ by the anesthetists $(p<0.001)$. The median total performance score was 2 for the pediatricians versus 12.3 for the anesthetists $(p<0.001)$. The median number of complications (see also Table 2 ) was 2.0 for the pediatricians versus zero for the anesthetists $(p<0.001)$. Pediatricians scored a significantly lower end-assessment grade for the total procedure with a median score of 3.5 versus 7.3 for the anesthetists $(p<0.001)$.

For the child manikin, 8 (15.4\%) pediatricians were considered sufficiently able to perform the procedure, compared with $49(94.2 \%)$ of the anesthetists $(p<0.001)$.

Table 3 shows the distribution of self-perceived capability of performing ETI in comparison with the qualification given by the observers: in some participants, there is a discrepancy between their self-perceived capability and the assessed performance.

Intra-class correlation coefficient For the median total performance score, the observers had a very high agreement 

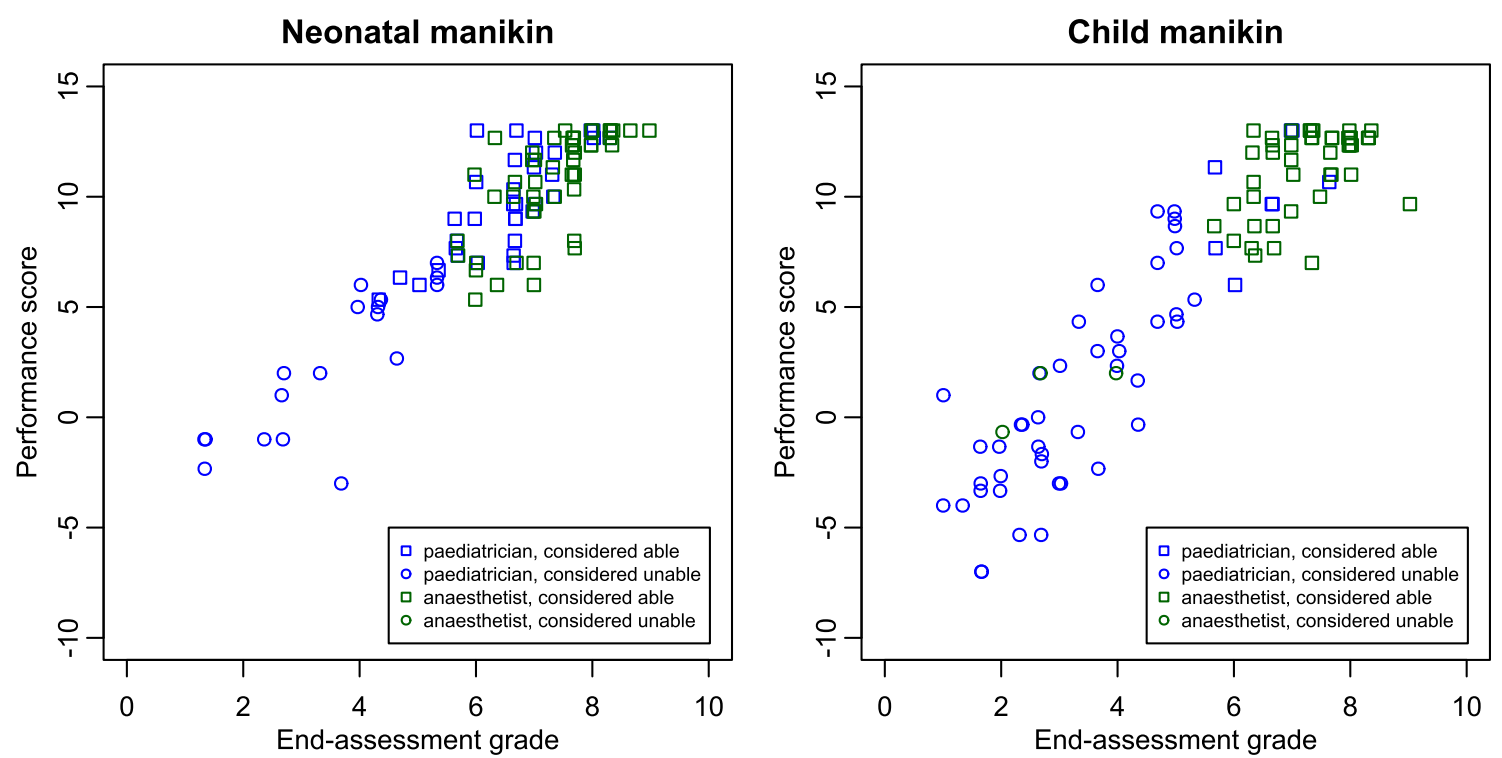

Fig. 3 Distribution of the end-assessment grade, the total performance score, and the consideration (in)sufficiently able to perform ETI on both manikins per specialty

(ICC $=0.983$ for the neonate, 0.989 for the child). There was complete agreement between the three observers for procedures on both manikins with respect to the complications $(\mathrm{ICC}=1.00)$. The observers' ICC was 0.81 and 0.86 respectively for the end-assessment grades of the participants on the neonatal and child manikin. The observers had $100 \%$ agreement on the ratings whether the participant was considered sufficiently able to perform the procedure.

\section{Discussion}

This study shows that anesthetists are better in performing ETIs on both neonatal and child manikin and perform significantly better on most components compared with pediatricians, resulting in a higher success rate and fewer complications. The main differences occurred at the stage of ETI, such as laryngoscopy and advancing the tube into the glottis and trachea. Although most of us would expect these findings, no study has ever been published, as far as we know, that compared the intubation skills of pediatricians with those of anesthesiologists in a standardized setting. Although there was no significant difference between both groups of specialists in successful neonatal ETI (i.e., tube in larynx through vocal cords), the performance of the total procedure by the anesthetists was significantly better on all other components, expecting fewer complications.

The majority of the Dutch pediatric and anesthetic participants still believed that pediatricians should perform the emergent neonatal intubation: most probably based on a historical basis. Yet, the anesthetist appears to be the most qualified person to carry out this procedure in general hospitals. Studies have shown that 50-60 ETI real-life procedures need to be conducted to achieve a $90 \%$ success rate in controlled settings $[13,22,25]$. However, $18 \%$ of providers still require assistance after 80 intubations [13]. These numbers will never be achieved by pediatricians in (Dutch) general hospitals. The exposure of pediatric residents to ETI is very low as well. In the Netherlands, neonatal and pediatric ETI is not an obligatory

Table 2 ETI complications on the neonatal and child manikin stratified by specialty

\begin{tabular}{|c|c|c|c|c|}
\hline & & Pediatricians $(n)$ & Anesthetists $(n)$ & $p$ value \\
\hline \multirow[t]{2}{*}{ Blade between vocal cords } & Neonate & $16(30.8 \%)$ & $9(17.3 \%)$ & 0.108 \\
\hline & Child & $25(48.1 \%)$ & $8(15.4 \%)$ & $<0.001$ \\
\hline \multirow[t]{2}{*}{ Tube in esophagus } & Neonate & $8(15.4 \%)$ & $0(0.0 \%)$ & 0.060 \\
\hline & Child & $21(42.0 \%)$ & $3(12.5 \%)$ & $<0.001$ \\
\hline \multirow[t]{2}{*}{ Transferring laryngoscope (form one hand to the other) } & Neonate & $16(30.8 \%)$ & $1(1.9 \%)$ & $<0.001$ \\
\hline & Child & $19(38.0 \%)$ & $2(3.8 \%)$ & $<0.001$ \\
\hline \multirow{2}{*}{$\begin{array}{l}\text { Incorrect cuff placement } \\
\text { (cuff between vocal cords) }\end{array}$} & Neonate & - & - & - \\
\hline & Child & $3(9.7 \%)$ & $0(0.0 \%)$ & 0.114 \\
\hline
\end{tabular}


Table 3 Self-perceived capability of performing ETI on neonates and children by pediatricians versus anesthetists, in comparison with their performance on a neonatal and child manikin

\begin{tabular}{|c|c|c|c|c|}
\hline & \multicolumn{2}{|l|}{ Neonate } & \multicolumn{2}{|l|}{ Child } \\
\hline & Pediatricians & Anesthetists & Pediatricians & Anesthetists \\
\hline Aware of competency & $42.3 \%$ & $67.3 \%$ & $0.0 \%$ & $94.5 \%$ \\
\hline Not aware of competency & $23.1 \%$ & $32.7 \%$ & $15.7 \%$ & $0.0 \%$ \\
\hline Aware of lack of competency & $5.8 \%$ & $0.0 \%$ & $74.5 \%$ & $0.0 \%$ \\
\hline Unaware of lack of competency & $28.8 \%$ & $0.0 \%$ & $9.8 \%$ & $5.8 \%$ \\
\hline
\end{tabular}

"entrustable professional activity" anymore during residency training $[5,10,23]$. Studies on neonatal ETI by pediatric residents show a low success rate of $20-26 \%$ [6, 12, 15, 19]. This lack of exposure to ETI cannot simply be replaced by a simulation-based manikin training, since this training is not a guarantee for successful skills in the acute care setting $[4,8]$.

Although Dutch general anesthetists also have limited exposure in performing neonatal ETI in particular, in general, they are far more expert in performing ETI procedures. They are highly skilled in airway management including the avoidance of potential serious complications. This makes them the most suitable persons to perform ETI in neonates and children in acute care settings.

There are countries, like the UK, that have made a national agreement that the anesthetist performs ETI in all neonates and children that require an emergent ETI. In our opinion, there is an urgent need to make this agreement in those countries that did not make this agreement yet. Our finding that quite a large number of the anesthetists is not aware of their own competence in performing neonatal ETI and one quarter of the pediatricians rated themselves capable to perform neonatal ETI while they were not skilled enough makes it unlikely that both occupational groups can make these agreements at hospital level. The national societies of anesthesia and pediatrics must take the lead and the responsibility to come to national agreements and directives.

\section{Facilitation of change}

Although the anesthetist is the most skilled person to perform the emergent ETI, the implementation of new national agreements on this subject might not be easy. Anesthetists have a low self-perceived competence in performing neonatal ETI and according to the results in the survey, a minority of the anesthetists stated that they should be responsible for the ETI in neonates. The restraint of some anesthetists to perform an emergency neonatal ETI is probably due to the difficulty to maintain neonatal ETI skills in general hospitals since there is a lack of regular on-site ETIs in neonates.

To address this problem, (1) an extensive, advanced pediatric airway management training course should be developed at a national level for general anesthetists/residents in anesthesia, and be mandatory both during residency and for re-registration. To maintain adequate skill, attendance at the national courses and local skill training programs and assessments are needed since, over time, there is significant decay in skills when not frequently used or refreshed [16, 26], especially in the field of neonatal intubation. (2) Tertiary care centers do have to play a key role in facilitating regional training facilities to enable anesthetists from general hospitals to remain skilled in and confident on neonatal ETI. (3) It is of no use to train general pediatricians in performing ETI since they cannot gain the practical airway-management experience needed to adequately perform ETI. Instead, there should be developed a national course for pediatricians/residents in pediatrics to obtain and maintain the skills in non-invasive maneuvers to guarantee a free airway, mask-and-bag ventilation and the introduction of a supraglottic airway device. Also, this course should be mandatory during residency and for re-registration in pediatrics.

\section{Limitations}

Literature shows that skills learned on manikins are not absolute guarantee for success in real-life situations [4, 8]. However, for this study, we had to use manikins, since it was impossible to perform the meticulous study in vivo on neonates and children. This was the best study design we could conceive to compare the two groups of medical specialists in a precise standardized setting. The restrictions given by the manikins were the same for both groups. Our study fulfills partially Kane's validity framework concerning the assessment strategy that was used to assess the ETI performance. Concerning the data evaluating "scoring," we tried to achieve a form of inter-item correlation by comparing the objective score (total performance score) with the subjective scores (end-assessment rating and an assessment) of the observers, to see if high total performance scores did correlate with high subjective scores and vice versa, which was the case. Besides this, we tested the inter-rater reliability, which was strong. With respect to the "generalizability," the overall inter-station reliability was high since the test stations were similar during all test situations. The materials used were identical during all tests and identical to the materials used in daily practice. The same introduction and instructions were given to the participant by the same instructors in all test situations. The data evaluating "extrapolation and implications" could not be executed. We 
did not correlate assessment scores by clinical errors or failure in practice since the participants were not tested in practice. In summary, we suggest the evidence for scoring and generalization supports the use of our simulation-based assessment strategy as a reflection of ETI performance in a simulated setting. However, in the extrapolation to daily practice, evidence is lacking, since test scores were not correlated to real clinical performances - mainly due to lack of clinical exposure by the participants. However, performing ETI on manikins is expected to be easier than performing ETI in practice, in particular in acute care settings. We therefore hypothesize that low assessment scores in the manikin model reflect low real-life ETI performance.

\section{Conclusions}

With this study, we determined that anesthetists are more successful and better qualified in intubating neonates and children compared with pediatricians in a simulated setting. Clear agreements between these specialties need to be made urgently and translated in concrete national and local protocols with the main appointment that, in general, the anesthetist will perform ETI on neonates and children in acute care settings.

Authors' contributions Sam J van Sambeeck conceptualized and designed the study, executed the study and data collection and analysis, drafted the initial and final manuscript, and approved the final manuscript as submitted.

Sander MJ van Kuijk helped conceptualizing and designing the study with special attention to the statistical analyses and the figures, critically reviewed the manuscript, and approved the final manuscript as submitted.

Boris W Kramer contributed with his expertise on this subject, helped conceptualizing the study, supervised data collection and intubation-video ratings as observer, critically reviewed and revised the manuscript, and approved the final manuscript as submitted.

Petronella M Vermeulen contributed with his expertise on this subject, helped conceptualizing the study, supervised data collection and intubation-video ratings as observer, critically reviewed and revised the manuscript, and approved the final manuscript as submitted.

Gijs D Vos contributed with his expertise on this subject, helped conceptualizing the study, made the adjustments on the laryngoscopes, supervised data collection and intubation-video ratings as observer, critically reviewed and revised the manuscript, and approved the final manuscript as submitted.

All authors approved the final manuscript as submitted and agree to be accountable for all aspects of the work.

\section{Compliance with ethical standards}

Conflict of interest All authors declare that they have no conflict of interest.

Ethical approval This article does not contain any studies with human participants or animals performed by any of the authors.

Informed consent Informed consent was obtained from all individual participants included in the study.
Open Access This article is distributed under the terms of the Creative Commons Attribution 4.0 International License (http:// creativecommons.org/licenses/by/4.0/), which permits unrestricted use, distribution, and reproduction in any medium, provided you give appropriate credit to the original author(s) and the source, provide a link to the Creative Commons license, and indicate if changes were made.

\section{References}

1. Andersen LW, Raymond TT, Berg RA, Nadkarni VM, Grossestreuer KT et al (2016) Association between tracheal intubation during pediatric in-hospital cardiac arrest and survival for the American Heart Association's Get With The Guidelinesresuscitation investigators. JAMA 316:1786-1797. https://doi. org/10.1001/jama.2016.14486

2. Barber CA, Wyckoff MH (2006) Use and efficacy of endotracheal versus intravenous epinephrine during neonatal cardiopulmonary resuscitation in the delivery room. Pediatrics 118:1028-1034. https://doi.org/10.1542/peds.2006-0416

3. Bernhard M, Mohr S, Weigand MA, Martin E, Walther A (2012) Developing the skill of endotracheal intubation: implication for emergency medicine. Acta Anaesthesiol Scand 56:164-171. https://doi.org/10.1111/j.1399-6576.2011.02547.x

4. Bradley JS, Billows GL, Olinger ML, Boha SP, Cordell WH, Nelson DR (1998) Prehospital oral endotracheal intubation by rural basic emergency medical technicians. Ann Emerg Med 32:26-32

5. Downes KJ, Narendran V, Meinzen-Derr J, McClanahan S, Akinbi HT (2012) The lost art of intubation: assessing opportunities for residents to perform neonatal intubation. J Perinatol 32:927-932. https://doi.org/10.1038/jp.2012.17

6. Falck AJ, Escobedo MB, Baillargeon JG, Villard LG, Gunkel JH (2003) Proficiency of pediatric residents in performing neonatal endotracheal intubation. Pediatrics 112:1242-1247

7. Fiadjoe JE, Nishisaki A, Jagannathan N, Hunyady AI, Greenberg RS, Reynolds PI, Matuszczak ME, Rehman MA, Polaner DM, Szmuk P, Nadkarni VM, McGowan FX Jr, Litman RS, Kovatsis PG (2016) Airway management complications in children with difficult tracheal intubation from the Pediatric Difficult Intubation (PeDI) registry: a prospective cohort analysis. Lancet Respir Med 4: 37-48. https://doi.org/10.1016/S2213-2600(15)00508-1

8. Finan E, Bismilla Z, Campbell C, LeBlanc V, Jefferies A, Whyte HE (2012) Improved procedural performance following a simulation training session may not be transferable to the clinical environment. J Perinatol 32:539-544. https://doi.org/10.1038/jp.2011.141

9. Foglia EE, Ades A, Napolitano N, Leffelman J, Nadkarni V, Nishisaki A (2015) Factors associated with adverse events during tracheal intubation in the NICU. Neonatology. 108:23-29. https:// doi.org/10.1159/000381252

10. Gozzo YF, Cummings CL, Chapman RL, Bizzarro MJ, Mercurio MR (2010) Who is performing medical procedures in the neonatal intensive care unit? J Perinatol 31:206-211. https://doi.org/10. 1038/jp.2010.121

11. Hatch LD, Grubb PH, Lea AS, Walsh WF, Markham MH, Whitney GM, Slaughter JC, Stark AR, Ely EW (2016) Endotracheal intubation in neonates: a prospective study of adverse safety events in 162 infants. J Pediatr 168:62-6.e6. https://doi.org/10.1016/j.jpeds.2015. 09.077

12. Haubner LY, Barry JS, Johnston LC, Soghier L, Tatum PM, Kessler D, Downes K, Auerbach M (2013) Neonatal intubation performance: room for improvement in tertiary neonatal intensive care units. Resuscitation 84:1359-1364. https://doi.org/10.1016/j. resuscitation.2013.03.014 
13. Katz SH, Falk JL (2001) Misplaced endotracheal tubes by paramedics in an urban emergency medical services system. Ann Emerg Med 37:62-64

14. Konrad C, Schüpfer G, Wietlisbach M, Gerber H (1998) Learning manual skills in anesthesiology: is there a recommended number of cases for anesthestic procedures? Anesth Analg 86:635-639

15. Leone TA, Rich W, Finer NN (2005) Neonatal intubation: success of pediatric trainees. J Pediatr 146:638-641

16. Mohammad A, Branicki F, Abu-Zidan FM (2014) Educational and clinical impact of Advanced Trauma Life Support (ATLS) courses: a systematic review. World J Surg 38:322-329. https://doi.org/10. 1007/s00268-013-2294-0

17. Mulcaster JT, Mills J, Hung OR, MacQuarrie K, Law JA, Pytka S, Imrie D, Field C (2003) Laryngoscopic intubation - learning and performance. Anesthesiology. 98:23-27

18. Neumar RW, Otto CW, Link MS, Kronick SL, Shuster M, Callaway $\mathrm{CW}$ et al (2010) Part 8 adult advanced cardiovascular life support. 2010 American Heart Association Guidelines for cardiopulmonary resuscitation and emergency cardiovascular care. Circulation. 122: 729-767

19. O'Donnell CP, Kamlin CO, Davis PG, Morley CJ (2006) Endotracheal intubation attempts during neonatal resuscitation: success rates, duration, and adverse effects. Pediatrics. 117:e16-e21

20. Pediatric Intensive Care Evaluation (PICE) (2013) Dataregistrationproject of Dutch paediatric intensive care units. http://www.pice.nl/dataset.htm. Accessed 31 May 2013

21. Perlman JM, Risser R (1995) Cardiopulmonary resuscitation in the delivery room: associated clinical events. Arch Pediatr Adolesc Med 149:20-25
22. Timmermann A, Russo SG, Eich C, Roessler M, Braun U, Rosenblatt WH, Quintel M (2007) The out-of-hospital esophageal and endobronchial intubations performed by emergency physicians. Anesth Analg 104:619-623

23. Toekomstbestendige Opleiding Pediatrie (2017) TOP 2020 (deel II) Het curriculum van de opleiding tot kinderarts. Projectgroep TOP2020. Uitgave van de Nederlands Vereniging voor Kindergeneeskunde. https://michaelgroeneweg.files.wordpress. com/2015/12/top2020-deel-2.pdf. Accessed 30 December 2017

24. Van Sambeeck SJ, Martens SJ, Hundscheid T, Janssen EJ, Vos GD (2015) Dutch paediatrician's opinions about acute care for critically ill children in general hospitals. Eur J Pediatr 174:607-613. https:// doi.org/10.1007/s00431-014-2439-7

25. von Goedecke A, Herff H, Paal P, Dörges V, Wenzel V (2007) Field airway management disasters. Anesth Analg 104:481-483

26. Woollard M, Whitfield R, Newcombe RG, Colquhoun M, Vetter N, Chamberlain D (2006) Optimal refresher training intervals for AED and CPR skills: a randomised controlled trial. Resuscitation 71: 237-247. https://doi.org/10.1016/j.resuscitation.2006.04.005

Publisher's note Springer Nature remains neutral with regard to jurisdictional claims in published maps and institutional affiliations.

\section{Affiliations}

\section{Sam J. van Sambeeck ${ }^{1}$ - Sander M. J. van Kuijk ${ }^{2}$ - Boris W. Kramer ${ }^{1} \cdot$ Petronella M. Vermeulen $^{3} \cdot$ Gijs D. Vos ${ }^{1}$}

Sander M. J. van Kuijk

sander.van.kuijk@mumc.nl

Boris W. Kramer

b.kramer@maastrichtuniversity.nl

1 Department of Pediatrics, Maastricht University Medical Centre, P. Debyelaan 25, P.O. Box 5800, 6202 AZ Maastricht, The Netherlands

2 Department of Clinical Epidemiology and Medical Technology Assessment, Maastricht University Medical Centre, P. Debyelaan 25, P.O. Box 5800, 6202 AZ Maastricht, The Netherlands
Petronella M. Vermeulen

petronella.vermeulen@mumc.nl

Gijs D. Vos

gijs.vos@hotmail.com

3

Department of Anesthesiology, Maastricht University Medical

Centre, P. Debyelaan 25, P.O. Box 5800, 6202

AZ Maastricht, The Netherlands 